rev.relac.int.estrateg.segur.9(2):119-141,2014

\title{
TERRITORIO Y ESTADO. CAMBIOS Y DESAFÍOS EN LA GLOBALIZACIÓN DEL DERECHO•
}

\section{William Guillermo Jiménez ${ }^{* *}$}

\section{RESUMEN}

La globalización está ejerciendo una fuerte presión sobre el Estado-nación como unidad básica de producción y aplicación del derecho, desafiando así las tradicionales fronteras temporales, espaciales y conceptuales del mismo. El objetivo de este trabajo es establecer los efectos que el proceso de globalización del derecho genera en la relación entre Estado y territorio. Es un estudio cualitativo que se realiza mediante las técnicas de la revisión documental, las entrevistas, la observación directa y el análisis de casos, recurriendo tanto a fuentes primarias como secundarias. Se concluye que, si bien el Estado-nación se ha debilitado en

Versión para artículo del informe final de investigación, titulado "La globalización del Derecho - Impactos y consecuencias actuales en la relación Estado y territorio", del Grupo Estado, Derecho y Territorio, Bogotá, Universidad Libre.

** PhD en Ciencias Políticas, abogado, administrador público, especialista en Derecho Administrativo, especialista en Desarrollo Regional; estudios postdoctorales en derecho. Profesor titular Escuela Superior de Administración Pública y Universidad Libre. Correo electrónico: wjimen@yahoo.com.mx,willjime@esap.edu.co. 
sus elementos clásicos, este sigue siendo importante y puede asumir tres tipos de rol frente a la globalización (Estado bisagra, Estado antisistema y Estado garantista); en cuanto a la relación jurídica entre Estado y territorio, se observa una superación del enfoque del territorio como ámbito de jurisdicción estatal, aunque, por otro lado, hay una exacerbación del territorio entendido como elemento esencial del Estado.

Palabras clave: Globalización del derecho, Estado-nación, territorio, tribunales internacionales, pluralismo normativo.

\title{
TERRITORY AND STATE - CHANGES AND CHALLENGES IN THE GLOBALIZATION OF LAW
}

\begin{abstract}
Globalization is making strong pressure on the Nation-state as the prime unit of creation and application of law, challenging traditional temporal, spatial and conceptual boundaries thereof. The aim of this paper is to establish the effects that the process of globalization of law creates in the relationship between state and territory. This is a qualitative study performed by the techniques of documentary review, interviews, direct observation and case analysis, using both primary and secondary sources. It concludes that although the Nation-state has weakened in its classic elements, it remains important and can assume three types of function against globalization (hinge state, anti-system state and guarantor state); concerning the legal relationship between state and territory, it is noted an upgrading of the idea of territory as area of state jurisdiction, although, on the other hand, there is a stress of territory understood as an essential element of the state.
\end{abstract}

Keywords: Globalization of law, Nation-state, territory, international courts, normative pluralism.

\section{TERRITÓRIO E ESTADO - MUDANÇAS E DESAFIOS NA GLOBALIZAÇÃO DO DIREITO}

\section{RESUMO}

A globalização está a exercendo uma forte pressão sobre o Estado-nação como unidade básica de produção e aplicação do direito, desafiando dessa maneira as tradicionais fronteiras temporais, espaciais e conceituais do mesmo. O objetivo deste trabalho é estabelecer os efeitos que o processo de globalização gera na relação entre Estado e território. É um estudo qualitativo realizado por meio de técnicas de revisão documentária, entrevistas, observação direta e da análise de casos, utilizando tanto as fontes primárias como secundárias. Conclui-se que, embora o Estado tenha enfraquecido em seus elementos clássicos, ainda é importante e pode assumir três tipos de papéis diante da globalização (Estado dobradiça, Estado antissistema e Estado 
garante); sobre a relação jurídica entre o Estado e o território, observa-se uma superação da abordagem de território como área de jurisdição do Estado, ainda que, por outro lado, exista uma exacerbação do território tratado como elemento essencial do Estado.

Palavras-chave: Globalização do Direito, Estado-nação, território, tribunais internacionais, pluralismo normativo.

\section{INTRODUCCIÓN}

Dos fallos recientes de tribunales internacionales en 2013 llaman la atención por sus dispares resultados, pese a que en ambos casos sus decisiones tenían carácter vinculante para los Estados implicados. En un caso, el Tribunal Internacional de Justicia de la Haya decidió un conflicto fronterizo entre Colombia y Nicaragua, favoreciendo a este último; sin embargo, el gobierno colombiano manifestó que tal fallo era inaplicable, argumentando que la Constitución Política colombiana solo permite que los límites fronterizos del país sean modificados mediante tratados internacionales (la Corte Constitucional colombiana avaló tal postura). En otro caso, el Tribunal Europeo de Derechos Humanos, atendiendo un recurso de una mujer condenada por delitos de terrorismo en España, decidió que se estaban violando los derechos a la igualdad y no discriminación de esa persona en la aplicación del beneficio de excarcelación; el Estado español cumplió el fallo y dejó en libertad a la mujer, pese al repudio generalizado.

Estos dos casos demuestran conflictos entre el Estado-nación y la tendencia globalizadora del derecho. Pareciera que en materia de defensa del territorio como elemento del Estado, los Estados tratan de privilegiar su soberanía territorial por encima de los compromisos internacionales; por otro lado, en temas de derechos humanos se demuestra que la soberanía nacional cede ante el derecho globalizado, el cual asume un carácter desterritorializado.

Durante las últimas 4 centurias el Estado se convirtió en el ente monopolizador de los siguientes procesos: a) El monopolio del uso de la fuerza sobre una población asentada en un territorio delimitado; b) El monopolio de la producción normativa de carácter jurídico o vinculante; c) El monopolio de la representación del interés general al interior, y la representación estatal al exterior como sujeto privilegiado del derecho internacional. Consecuentemente, la doctrina jurídica ha reconocido tres elementos básicos para la existencia del Estado-nación: 1) Un pueblo o nación; 2) Un territorio o espacio físico, y 3) Un poder político soberano. Finalmente, para el logro de sus fines o cometidos ontológicos y axiológicos, el Estado-nación ha ejercido de manera exclusiva y excluyente ciertas funciones básicas que implican el ejercicio de potestad o autoridad: funciones públicas legislativa, administrativa y jurisdiccional.

La evidencia reciente sobre la globalización, parece demostrar que el Estado nacional está perdiendo el monopolio en la producción del derecho y tendríamos así el paso de un derecho eminentemente "estado céntrico" a un derecho de corte "global céntrico", o lo que algunos 
han denominado "pluralismo normativo" (Wolkermer, 2006; Ramírez, s. f.). De igual manera, el papel del territorio como ámbito de aplicación de los ordenamientos jurídicos está afectado con lo que se conoce como "desterritorialización" o "nuevas espacialidades" más allá del territorio (Agnew, 2008).

En principio se tendría una doble dimensión del asunto: por un lado, la pregunta acerca de cuál es la naturaleza y papel que la globalización le reserva al Estado-nación; de otro lado, en la medida en que el rol y funciones del Estado en la globalización se transforman, cabría preguntarse qué le depara al territorio no solo en su relación con el Estado, sino además en su papel tradicional de contenedor del derecho. El problema de investigación es el siguiente:¿De qué manera influye la globalización del derecho en la relación entre Estado y territorio, en especial en la regulación jurídica del territorio establecida desde el paradigma del Estado-nación?

El objetivo general del artículo es establecer las consecuencias y efectos que el proceso de globalización del derecho genera en la relación entre Estado y territorio, debido al aparecimiento de nuevos tipos de regulación jurídica tendencialmente desterritorializadas y no propiamente estatales. Los objetivos específicos son los siguientes: a) Aclarar qué papel cumple el Estado-nación en el contexto de la globalización; b) Determinar las implicaciones que tiene para el territorio la desterritorialización de la regulación jurídica; c) Analizar el rol que cumplen los tribunales internacionales de justicia en la producción y regulación jurídica; d) Elucidar los cambios en el derecho constitucional y administrativo, como resultado del proceso de globalización.

\section{GLOBALIZACIÓN, ESTADO Y TERRITORIO}

Sobre conceptos y debates acerca de la globalización, la globalización del derecho, los cambios del Estado, el constitucionalismo y el territorio, ya se habían publicado avances del proyecto, baste aquí recapitular la posición frente a determinados asuntos.

La globalización es un fenómeno complejo y la vez una elaboración intelectiva o marco teórico-explicativo sobre el mismo. El proceso globalizador puede ser inevitable desde el punto de vista fáctico, pero hay múltiples enfoques acerca de sus ventajas y desventajas, es decir, no puede haber un modelo único o dominante sobre la globalización (Martínez, 2010; Santos, 2002). A propósito, recuerda Twining (2003: 119-120) "La literatura acerca de la globalización es casi tan confusa como los procesos que busca interpretar... Hay teorías de inspiración marxista, las hay de corte posmoderno, las hay que celebran el triunfo del libre mercado, las de corte religioso y libertario...". A riesgo de simplificación, se pueden establecer algunos de sus rasgos más característicos:

- $\quad$ Es un proceso o conjunto de procesos complejos que tienen en común la superación del Estado-nación como actor privilegiado en las relaciones transnacionales. 
- Implica interdependencia e interacción (entre mercados, Estados, empresas, organizaciones y sujetos).

- Señala intercambios y flujos continuos alrededor del globo (tanto de personas, bienes, capitales y servicios).

- Se manifiesta en los campos económico, cultural, político, poblacional, tecno-científico y jurídico; cada uno de estos campos con su propia lógica y racionalidad (por ello se habla de varias globalizaciones).

- Genera procesos de desterritorialización y, a su vez, de relocalización espacial (glocalización).

- Es un fenómeno asimétrico, es decir, que no genera los mismos beneficios y ventajas para todos, conllevando más bien a una concentración inusual de capital (por ello tiene defensores y detractores).

- Es tanto un proceso de homogenización (estandarización mundial) como también de diferenciación (búsqueda de identidades regionales, étnicas y locales).

- La globalización actual inicia a comienzos de los setenta con una nueva división internacional del trabajo (internacionalización de capitales) y se sustenta en la actualidad con la revolución científica y tecnológica, especialmente los desarrollos de las TIC's.

En las diferentes aproximaciones sobre derecho y globalización, el común denominador es la consideración acerca del desafío que representa la globalización para el derecho, dado que el derecho moderno se ha desarrollado bajo el paradigma del Estado- nación; es decir, el derecho se ve afectado al afectarse el Estado-nación. Al respecto, los trabajos de Borja y Castells (1998) señalan que con la globalización pareciera que el Estado-nación se ha tornado muy pequeño para proteger a sus ciudadanos de los impactos externos, pero a la vez muy grande para atender las necesidades concretas de sus habitantes; en igual sentido, expresa Bell (2000, p.25) "La nación se hace no sólo demasiado pequeña para solucionar los grandes problemas, sino también demasiado grande para arreglar los pequeños". A nivel del derecho constitucional, por ejemplo, se alude a la crisis del modelo estatal westfaliano (llamado así por el Tratado de Westfalia de 1648), en tres aspectos: a) El principio democrático de la soberanía popular; b) El principio liberal de declaración de derechos y la separación de poderes públicos; c) El principio de supremacía constitucional (Tajadura, 2004).

De otro lado, se han señalado algunas repercusiones de la globalización sobre los tres elementos básicos del Estado moderno: la población, el territorio y el poder soberano. La idea de un conglomerado social, asentado sobre un territorio determinado y sometido un poder político soberano, es todavía una definición comúnmente aceptada por el derecho (Naranjo, 2003). Sin embargo, hoy en día estos elementos ofrecen dificultades para dar una explicación satisfactoria 
como requisitos esenciales del Estado. Las migraciones y alta movilidad de la población, las regulaciones transfronterizas y la erosión de la soberanía nacional, son apenas ejemplos de los nuevos fenómenos.

En lo que concierne específicamente al territorio, la superación de las fronteras territoriales es un hecho que se evidencia con los siguientes fenómenos globalizados: a) El ascenso de la gobernanza de sistema global que produce "legislación mundial", concretamente la Organización de Naciones Unidas - ONU; b) La mayor importancia de acuerdos regionales o supraestatales como UE, CAN, OTAN, OCDE, etc., que producen tanto derecho internacional como derecho de integración; c) El ascenso de organizaciones no estales que producen su propio derecho, especialmente en áreas del derecho comercial, tales como la Cámara Internacional de Comercio o el Instituto Internacional de Derecho Privado, y que generan específicas regulaciones (ICOTERMS, lex mercatoria, arbitraje internacional, etc.); d) El ascenso vertiginoso del cyberlaw, o derecho del internet, es otro desafío al clásico derecho territorial: "Here, legal regulations have become global and policentric, thus raising cuestions as to the locus and legitimacy of the legislative body, the nature of the relevant legal communities, etc" (VU Amsterdam, 2013, p.2).

En este contexto aparecen las nociones de desterritorialización y relocalización. La primera significa que los ámbitos de interacción ya no son físicos o delimitados geográficamente; existe una diferencia entre "lugar" (de orden físico o material) y "espacio" (de orden intelectivo o virtual). La globalización genera procesos que se ubican muchas veces en "espacios virtuales" que no se corresponden con una ubicación o lugar físico palpable, pero que requieren de un nivel de regulación puesto que se trata de interacciones humanas potencialmente generadoras de derechos y obligaciones. Por su parte, la relocalización significa la ubicación física de actividades globalizadas dentro de un determinado territorio geográfico, porque lo global se gestiona en lo local, o porque los problemas son globales, las soluciones son locales; esto ha generado toda una serie de interrogantes sobre la gestión y los ámbitos de regulación jurídica de dichas actividades relocalizadas (por ejemplo, las laborales y comerciales), además de los impactos ambientales y sociales muchas veces perniciosos.

De lo anterior se desprende el tema de la extraterritorialidad de la jurisdicción. Lo que se evidencia es un cambio para la regulación del territorio y de las actividades que sobre él se desarrollan. Antes la jurisdicción estaba determinada por el territorio y esto no ha desaparecido, pues el lugar (del domicilio, de los hechos, del contrato, del daño...) sigue siendo central para determinar tanto la ley aplicable, como el juez de conocimiento, pero la territorialidad está decayendo en importancia como un principio jurisdiccional. Hoy las reglas para establecer la jurisdicción son más amplias, flexibles o funcionales que lo estrictamente territorial, como por ejemplo, los test sobre los efectos intencionales, el nivel de interacción en las web pages, la accesibilidad dirigida, la objetiva conexión doméstica o el domicilio virtual, son usados para resolver casos de jurisdicción en internet (Jaeger-Fine, 2013; Manrique, 2011; Reindenberg, et. al, 2013). 


\section{TERRITORIO Y ESTADO. SU RELACIÓN JURÍDICA}

Es necesario conocer la relación jurídica entre Estado y territorio, para establecer luego el impacto de la globalización del derecho en dicha relación. A este respecto, en la tradición del derecho político se han desarrollado tres grandes planteamientos: 1) El territorio como objeto o propiedad; 2) El territorio como esencia; y 3) El territorio como ámbito jurisdiccional del Estado (Herrero, 1983; De Blas, 1981).

En el primer caso, el territorio se considera una cosa sobre la que se tiene derecho de propiedad, tal como la ejerce una persona sobre un bien (derecho real o de dominio). En este sentido, el Estado puede ceder, alquilar, vender, en fin, disponer de su territorio de igual manera como lo hace una persona sobre una cosa. Esta teoría ha tenido pocos adeptos en la doctrina debido a la consideración irrefutable que el territorio hace parte del mismo Estado, no es algo exterior a él y, por lo tanto, es esencia, no una cosa o res. Sin embargo, se han esgrimido diversos planteamientos para defender esta postura como por ejemplo, la doctrina de Jellinek y Michoud del imperium sobre el territorio; la doctrina del derecho real institucional de Dabin y Bordeau y, finalmente, la tesis del dominio inminente que emplea el concepto de soberanía (Naranjo, 2003).

En la segunda postura, el territorio se considera parte o esencia del Estado, es un componente de orden ontológico para el mismo, es parte de su ser: si falta el territorio falta el Estado; es como el cuerpo para la persona, pues una persona no puede existir sin un cuerpo. Aquí carecen de sentido los planteamientos sobre el dominio que pueda tener el Estado sobre el territorio, más bien hay un condicionamiento territorial de la vida del Estado, que genera la facultad de ejercer poder sobre las personas que viven dentro del territorio (imperium) y una relación de dominio público sobre ciertas partes del territorio, pero sin recurrir al derecho de propiedad. Adicionalmente, esta postura ofrece cuatro postulados:

- $\quad$ El postulado unidad se refiere a la unidad jurídica que se produce dentro del territorio, no a la continuidad material o física del terreno; por ejemplo, la Constitución colombiana rige tanto en el continente como en los territorios insulares, en su mar territorial y hasta en el segmento de órbita geoestacionaria (CP, arts.101, 310).

- La indivisibilidad se predica de la imposibilidad de libre parcelación del territorio por parte del soberano, como ocurría en las monarquías absolutas. Mantener la integridad del territorio es un fin esencial del Estado $\left(\mathrm{CP}\right.$, art. $\left.2^{\circ}\right)$.

- La impenetrabilidad expresa la cualidad excluyente y exclusiva del poder de un Estado sobre su territorio, lo cual lo obliga a rechazar cualquier intervención o agresión externa o tránsito de tropas extranjeras sin el debido consentimiento.

- La inalienabilidad indica la prohibición de la venta, cesión o disminución del territorio estatal, por parte de los gobernantes y autoridades públicas. 
Finalmente, en la tercera perspectiva el territorio no es un objeto sobre el que se tenga propiedad, como tampoco se le considera una parte constitutiva del Estado; es simplemente un límite geográfico del derecho del Estado o el espacio que limita el orden jurídico del Estado, es decir, su jurisdicción como ejercicio de su soberanía ${ }^{1}$. Uno de los defensores de esta idea es Kelsen, quien concibe al territorio "...exclusivamente, como el campo en que queda constreñido el orden jurídico creado por el Estado, evitando así conflictos con otros ordenamientos normativos" (De Blas, 1983: 89). Una de las consecuencias de esta postura ha sido el desarrollo del conocido principio de territorialidad de la ley, aunque, por otro lado, también obedezca a desarrollos del principio internacional de no intervención.

\section{PROCESAMIENTO DE LA INFORMACIÓN - FUENTES Y TÉCNICAS}

El estudio es de tipo correlacional, ya que se trata de señalar la manera como la globalización afecta otras variables como el Estado y el territorio; el enfoque metodológico es cualitativo, pues se aprecia el objeto de estudio desde el punto de vista de sus cualidades y características antes que una medición o cuantificación del fenómeno. Se utilizaron fuentes primarias y secundarias. Dentro de las primarias se encuentran la jurisprudencia, la normatividad y las personas entrevistadas, del siguiente modo: a) 26 textos de sentencias, fallos e interpretaciones prejudiciales de tribunales y cortes como el Tribunal de Justicia de la Comunidad Andina - TJCA, el Tribunal de Justicia de la Unión Europea - TJUE, el Tribunal Constitucional Federal Alemán, la Corte Internacional de Justicia, la Corte Constitucional colombiana, el Consejo de Estado colombiano; b) 20 textos de tratados, decisiones, constituciones, leyes, decretos, resoluciones y reglamentos provenientes del derecho internacional, del derecho comunitario y del derecho nacional; c) 7 personas entrevistadas.

Como fuentes secundarias, se acudió a una gran variedad de libros, artículos de revistas, documentos de Internet, tesis y ponencias que suman alrededor de 80 ítems diferentes.

Para obtener y generar la información desde las fuentes, se emplearon las técnicas de: 1) Análisis y revisión documental, para ello se realizaban las lecturas de los textos, se identificaban las ideas centrales y se elaboraba una reseña en la mayoría de los casos; 2) Observación directa, en especial sobre cuatro casos del TJCA y cuatro del TJUE en controversias entre el derecho nacional y el comunitario; 3) Entrevistas, se aplicó un instrumento a siete personas, 4 académicos y 3 funcionarios expertos; se realizó un análisis separadamente entre académicos y funcionarios. Los cuadros 1 y 2 sintetizan las respuestas ofrecidas.

1. Jurisdicción en sentido amplio, significa poder de Estado para: a) Prescribir normas (to prescribe, legislativo); b) Decidir conflictos (to adjudicate, judicial), c) Obligar cumplimiento de normas como de sentencias (to enforce, ejecutivo) (Kohl, 2010; Svantesson, 2007). Algunos consideran que el poder de los jueces para escuchar y decidir casos es "jurisdicción judicial" (Manrique, 2011). Obviamente, esto desborda el origen romano del término. 
Cuadro 1. Resultados entrevistas académicos.

\section{$\mathbf{N}^{0}$ pregunta}

Pregunta 1

¿Qué relación encuentra usted entre globalización y derecho?

Pregunta 2

¿Cuál sería el papel que asume el

Estado-nación dentro del proceso de globalización del derecho?

Pregunta 3

¿Qué cambios se podrían esperar en el derecho interno, debido a la globalización del derecho?

\section{Síntesis respuestas}

La actual globalización tiene un componente jurídico más allá de lo económico. Procesos globales impactan en derecho interno y este también trata de regular fenómenos. El Derecho asegura o regulariza la globalización.

El Estado-nación transforma y reacomoda su papel dentro de la globalización. No se prevé su desaparición; sin embargo, es evidente que cada vez está más limitado al ceder soberanía y debe aprender a actuar junto con nuevos actores y regulaciones globalizadas.

El derecho interno recibe directamente las influencias de las dinámicas externas; en este sentido, es menos autónomo. Unas de las características serán la hibridación, la multiculturalidad y la necesidad de armonización legislativa internacional.
Pregunta 4

¿Cuáles serían las áreas o campos del derecho más afectadas por la globalización?
En primer lugar el derecho económico-financiero; luego no hay claridad, pero se mencionan los $\mathrm{DDHH}$, el derecho público, el penal y el laboral.
Pregunta 5

¿Cómo afecta el proceso de globalización al Estado-nación en cuanto a la transferencia de poderes en organismos de carácter transnacional o de integración?

Pregunta 6

¿Considera usted que el territorio sigue siendo el elemento central que define la competencia jurídica del Estado-nación?

Pregunta 7

¿Considera usted que el territorio seguirá siendo un parte fundamental del Estado-nación?
En primer lugar, el Estado-nación pierde soberanía; en segundo lugar, se ven sometidos a intervención desde el exterior; esto conlleva a pérdida de representatividad política, pues las decisiones vienen de afuera. Una alternativa a este fenómeno serían los procesos de integración regional. También existe injerencia de organismos internacionales organizados al margen de los Estados.

No hay tendencia marcada; para dos profesores, el territorio sigue siendo muy importante; para los restantes, la globalización implica una pérdida de competencia jurídica que supera el marco territorial estatal. La deslocalización hace que el territorio ya no opere como límite jurisdiccional o competencial.

En general, el territorio seguirá siendo elemento esencial para el Estado; sin embargo, hay transformaciones en la relación territoriociudadano, y en la concepción física, la cual debe considerar la virtualización del territorio.

\section{Pregunta 8}

¿Sabe algo acerca de la llamada desterritorialización del derecho? ¿En qué consiste?
La mayoría entiende la desterritorialización del derecho como deslocalización de la producción jurídica en múltiples lugares con la participación de actores no tradicionales diferentes al Estado.

Fuente: Elaboración propia a partir de las entrevistas. 
Cuadro 2. Resultados entrevistas funcionarios.

\section{$\mathbf{N}^{0}$ pregunta}

\section{Síntesis respuestas}

Pregunta 1

¿Qué relación encuentra usted entre globalización y derecho?

Se coincide en que la relación es fuerte o estrecha. Es mediante el derecho que se construyen estructuras para la globalización. El derecho internacional ha cumplido esta función, pero debe adaptarse al cambio.

Pregunta 2

Existe cierta incertidumbre, pues si bien el Estado ha jugado ¿Cuál sería el papel que asume el un papel muy importante en el Derecho Internacional, hoy Estado-nación dentro del proceso de adquieren importancia actores particulares y la capacidad el globalización del derecho? Estado parece debilitarse.

Pregunta 3

El derecho interno es muy sensible; hay un reflejo de la ¿Qué cambio se podrían esperar en el derecho interno, debido a la globalización del derecho? globalización del derecho y las legislaciones internas se ajustan a parámetros internacionales, por ejemplo, comercio y negocios o asuntos penales.

Pregunta 4

¿Cuáles serían las áreas o campos del derecho más afectadas por la globalización?

Hay tendencia en otorgar prioridad al derecho comercial y al derecho internacional, aunque al final todas las áreas se vean afectadas.

Pregunta 5

¿Cómo afecta el proceso de globalización al Estado-nación en cuanto a la transferencia de poderes en organismos de carácter transnacional o de integración?
Lo afecta al limitar su soberanía y al generar más interdependencia. Esto es irreversible. Unos Estados sentirán mayores efectos que otros, pero podría entenderse que lo que se debilita del Estado con la cesión de soberanía se puede ganar con procesos de integración.

\section{Pregunta 6}

¿Considera usted que el territorio Aunque el territorio continúa siendo elemento esencial del Estado, sigue siendo el elemento central que se ha reconocido jurisdicción de autoridades internacionales al define la competencia jurídica del interior del territorio. Esto no ha sido fácil de entender y aceptar. Estado-Nación?

Pregunta 7

¿Considera usted que el territorio seguirá siendo una parte fundamental del Estado-nación?
Sí, el territorio sigue siendo importante. El desafío es cómo el cambio en la soberanía puede cambiar el concepto de territorio.

\section{Pregunta 8}

¿Sabe algo acerca de la llamada desterritorialización del derecho? ¿En Se desconoce el término y su significado. qué consiste?

Fuente: Elaboración propia a partir de las entrevistas realizadas. 


\section{ANÁLISIS DE LA INFORMACIÓN Y LOGRO DE OBJETIVOS}

Con todo lo recolectado se construyó un capítulo sobre estado del arte, un capítulo sobre marco teórico y jurídico, dos capítulos sobre los casos de la CAN y la UE y un capítulo sobre metodología y análisis de la información. A continuación, se realiza la triangulación desde las diferentes fuentes para dar respuesta a cada objetivo específico.

\subsection{PAPEL DEL ESTADO-NACIÓN EN EL CONTEXTO DE LA GLOBALIZACIÓN}

Sobre el rol del Estado-nación, tanto la revisión del estado del arte, las entrevistas y los casos analizados de la CAN y la UE demuestran, a todas luces, que el Estado ha cedido soberanía y competencias en la globalización. El punto neurálgico es establecer el rol que le espera al Estado-nación en ese contexto.

Un primer elemento común, que se infiere del estado del arte y la doctrina, es que el Estado no desaparecerá; partiendo de esta premisa, la pregunta será entonces, ¿Qué hará?, o ¿Qué papel desempeñará en el contexto de la globalización? Según la información obtenida en las entrevistas, el Estado se ha debilitado en la globalización, ya por la cesión de soberanía, ya porque han aparecido otros actores relevantes con los que debe contar. Sin embargo, no hay una respuesta clara frente al papel que el Estado-nación tiene frente a la globalización del Derecho, aunque se reconoce que aún desempeña un papel relevante en el derecho internacional.

De acuerdo con la revisión del estado del arte, el Estado puede variar su rol entre dos grandes escenarios: a) Un papel protagónico en el cual reafirma su soberanía e independencia frente a las tendencias de la globalización, y b) Un papel relegado y dependiente de las presiones globalizadoras, en especial de los consensos y políticas fijadas exteriormente (Ramírez, s. f.; Maestro, 2001; Tajadura, 2004; Gutiérrez, 2002). Este doble movimiento generaría una escala de Estados, unos más abiertos, otros más recelosos a los aires globalizadores.

Ahora bien, en los estudios de los casos jurídicos de la CAN y la UE, aunque centrados en el papel de los tribunales de justicia, se evidenció la prevalencia y aplicación del derecho internacional y del derecho de integración al interior de los Estados miembros; incluso, hubo casos en los cuales los Estados fueron sancionados por no aplicar el derecho comunitario, como sucedió con Francia. Lo anterior significa que efectivamente el Estado-nación ha cedido soberanía, y que esta cesión o traslado implica perder jurisdicción en las competencias trasladas: mientras el Estado pierde competencias, los organismos supraestatales ganan, en lo que parecería un juego de suma cero.

Sin embargo, se puede inferir que el Estado-nación es aún necesario e importante: 1) Para hacer posible la aplicación del propio derecho globalizado o comunitario (implementación de políticas públicas globales aplicadas localmente); 2) Porque aún conserva una importante 
tradición socio-cultural, histórica, política, económica y jurídica que, en el momento actual, la globalización no está en capacidad de sustituir; 3) Porque es el directo responsable de la provisión de bienes y servicios necesarios y esenciales para el bienestar de los habitantes (seguridad, servicios públicos sociales, infraestructuras, promoción del bienestar, etc.), materias sobre las cuales las organizaciones internacionales están lejos aún de asumir directamente.

\subsection{LAS IMPLICACIONES QUE TIENE PARA EL TERRITORIO LA DESTERRITORIALIZACIÓN DE LA REGULACIÓN JURÍDICA}

En primer lugar, el término desterritorialización del derecho, el cual supone una desconexión de la producción y la aplicación del derecho respecto del territorio físico, es poco conocido o poco teorizado y analizado; tal vez, el término deslocalización sea más popular. Esto se evidenció en las entrevistas y en la revisión documental. Se trata de una categoría académica antes que un vocablo de uso corriente en la práctica política, administrativa o profesional.

Según lo encontrado, el territorio ha dejado de ser, de manera exclusiva, el ámbito de competencia jurídica de un determinado Estado, es decir, que la doctrina jurídica que concebía al territorio como ámbito físico de jurisdicción de la autoridad y del derecho estatal debe ser revisada. En este sentido, el principio de territorialidad de la ley que comparte la misma significación sobre el territorio también se ve afectado. Lo anterior no significa que la localización o el lugar (del domicilio, de los hechos, del daño, del acceso, etc.) deje de ser relevante a la hora de establecer la ley aplicable y el juez que debe conocer, sino que estos criterios ya no obedecen a una automática o supuesta conexión con determinado territorio (Kohl, 2010; Svantesson, 2007).

Al respecto, los entrevistados reconocen que la globalización implica una pérdida de competencia jurídica que supera el marco territorial del Estado-nación, lo cual se evidencia en el reconocimiento de la jurisdicción de autoridades internacionales o externas. De otro lado, en la revisión de antecedentes se encontró que la globalización está desafiando las concepciones tradicionales sobre el derecho en sus límites espaciales, límites temporales y límites conceptuales (VU, 2013); en lo que toca con el territorio (ligado a un determinado Estado o distrito), este ha dejado de ser el criterio preponderante para establecer la jurisdicción y competencia para la generación y aplicación del derecho, como se indicó. Además, un importante sector de la doctrina hoy se pregunta si los principios básicos del derecho moderno se sostienen en la globalización, por ejemplo, las nociones de poder soberano, división de poderes y territorio (Maestro, 2001; Tajadura, 2004; Gutiérrez, 2002).

Finalmente, los análisis realizados sobre el papel de los tribunales internacionales de la CAN y la UE demuestran el desarrollo de un derecho comunitario o de integración, producido y aplicado dentro de un espacio físico que supera las fronteras de cada uno de los Estados miembros. Concomitante con el despegue de este derecho se encuentra un conjunto de organismos o autoridades supraestatales que, al recibir las competencias delegadas por los Estados, ejercen jurisdicción y competencia sobre el conjunto del territorio. Todo lo anterior, sin sumar las 
transacciones y relaciones jurídicas que se derivan del uso del internet como nuevo desafío para la noción tradicional de territorio, entendido tradicionalmente como el lugar de soporte físico de la actividad humana.

\subsection{EL ROL QUE CUMPLEN INSTITUCIONES INTERNACIONALES EN LA PRODUCCIÓN Y REGULACIÓN JURÍDICA, ESPECIALMENTE LOS TRIBUNALES INTERNACIONALES DE JUSTICIA}

Los cuatro casos analizados de la $\mathrm{CAN}^{2}$ y los cuatro de la $U E^{3}$ señalan que la jurisprudencia de los tribunales de justicia tiene una importancia cardinal para la aplicación uniforme y armónica del derecho comunitario y la desterritorialización del derecho en los procesos de integración regional. En los casos europeo y andino, dicha jurisprudencia es parámetro de interpretación obligatoria para los jueces nacionales en aquellos casos que resuelven controversias en las cuales están involucradas normas del derecho comunitario y la decisión a tomar no admite recurso alguno. Además, sus fallos tienen un carácter vinculante, garantizado por el tratado de creación respectivo en el cual los Estados miembros han transferido soberanía y ciertas competencias a otros órganos supraestatales. Estos tribunales no solamente resuelven recursos de nulidad de las normas comunitarias que posiblemente violen los tratados de creación o los derechos fundamentales sino que, además, imponen sanciones a los Estados miembros que incumplen el derecho comunitario. Por ello, estos organismos se convierten en una pieza fundamental dentro de los procesos de integración y de globalización del derecho.

Sin embargo, en el tema de la jerarquía normativa el asunto no es tan claro y contundente, pues frente a la constitución política de cada Estado miembro el asunto varía según la postura del respectivo tribunal constitucional (De Miguel, 2010). Así, en la medida en que el tribunal nacional enarbole argumentos basados en la tradición nacional, la soberanía popular y la participación de los ciudadanos en la constitución del poder político (y jurídico), la postura es a favor de la constitución como norma normarum y como ley fundamental; contrario sensu, en países pequeños sometidos históricamente a procesos de integración, de tránsito o de interacción con los vecinos, la posición es más relajada y proclive a aceptar el ordenamiento supranacional, como un orden superior, incluso por encima de la propia constitución. Los casos extremos de Alemania y Países Bajos ilustran lo anotado.

2. TJCA Interpretaciones Prejudiciales No. 28-IP-99 (caso Sáchica contra la Nación) No. 105-IP-2004 (caso PFIZER contra INVIMA). Consejo de Estado, Sección Primera, Sentencia de 27 de enero de 2000, radicación (4360), C. P., Santiago Urueta Ayola. Sentencia de 1 de agosto de 2002, radicación (6983), C. P., Gabriel Eduardo Mendoza; Sentencia de 4 de agosto de 2005, radicación (11001-03-24-000-1999-06015-01), C.P., Rafael Ostau de Lafont; Sentencia de 28de abril de 2011, radicación11001-03-24-000-2003-00532-01, C.P., Rafael Ostau de Lafont.

3. TJUE asunto C-121/07 (Comisión Europea contra Francia (Gran Sala - Diciembre 2008); asunto 383/09 Comisión Europea contra Francia (Sala Cuarta- Junio 2011); Asunto C-464/01- Sentencia de20 de enero de 2005 (Gruber v. Bay Wa AG Austria); Sentencia del TJUE (Gran Sala), de 16 de abril de 2013 (Italia y España contra el Consejo Europeo). 
En el caso andino, Colombia se acerca mucho más a la concepción alemana que a la holandesa, pues la Corte Constitucional ha sostenido que el derecho comunitario no hace parte del bloque de constitucionalidad (como tampoco del bloque de convencionalidad, a no ser que sean tratados sobre derechos humanos) y, por tanto, este derecho no es parámetro de control de otras normas siendo de una jerarquía igual a la de la ley; de esta manera queda abierta la posibilidad de que la Corte Constitucional colombiana realice un "control excepcional", similar al que reclama el Tribunal Constitucional alemán, en los casos en los cuales la justicia comunitaria no garantice un estándar mínimo de protección de derechos humanos o principios fundamentales ${ }^{4}$.

La situación del derecho de integración o derecho comunitario, luego del estudio realizado, revela dos facetas. La primera se refiere a las características especiales de prevalencia, efecto directo, aplicación inmediata y autonomía (Palomares, 2007; Blanco, 2012); sin embargo, una segunda cara presenta las limitaciones de este derecho, las cuales se pueden enunciar de la siguiente manera: a) Subordinación a principios superiores y derechos fundamentales establecidos en las constituciones de los países miembros. b) No hacen parte del bloque de constitucionalidad, a no ser que estén incluidos en tratados de derechos humanos; el derecho de integración o comunitario, al perseguir objetivos comerciales y de promoción del desarrollo económico (mercados comunes, uniones monetarias), no se fundamenta en tratados de derechos humanos. No es raro encontrar, entonces, que en Europa el tema de los derechos humanos haya sido encomendado al Tribunal Europeo de Derechos Humanos, y en el área andina al Tribunal Interamericano de Derechos Humanos, ambos independientes tanto de la UE como de la CAN. c) Excepcionalmente estarían sujetos al control de constitucionalidad por un tribunal nacional, cuando se considere que una norma comunitaria contraríe abiertamente los principios y derechos fundamentales establecidos en la constitución, y el tribunal internacional, encargado de su custodia, no haya hecho nada al respecto.

Los conflictos de competencias entre los tribunales constitucionales y los tribunales internacionales reflejan las disputas entre la concepción del derecho constitucional que tienen los primeros (el cual se ha desarrollado bajo los postulados del Estado-nación) y la concepción del derecho globalizado que tienen los segundos (Balaguer, 2007; De Miguel, 2010). Esto es evidente tanto en la revisión de los casos en la UE y la CAN, así como en las posiciones doctrinales encontradas en el estado del arte y en el marco teórico.

Entonces, hay en la actualidad una dificultad en la armonización de los postulados centrales del derecho constitucional aferrados todavía a una teoría del Estado y la constitución basados

4. BVerfGE (sentencia del Tribunal Constitucional Federal alemán) sobre el Tratado de Maastricht, 12 de octubre de 1993; BVerfGE sobre la resolución de ordenación del mercado del plátano,7 de junio 2000; BVerfGE sobre el Tratado de Lisboa, de 30 de junio de 2009. Para el caso colombiano, Sentencia de la Corte Constitucional colombiana C-231 de 1997, M.P. Eduardo Cifuentes.

TERRITORIO Y ESTADO. CAMBIOS Y DESAFÍOS EN LA GLOBALIZACIÓN DEL DERECHO 
a su vez en las ideas de pueblo o nación, territorio, poder soberano, separación de poderes y primacía de la constitución, entre otros, y el surgimiento de un derecho global que desafía precisamente aquellos postulados para enfatizar en otros tales como el pluralismo jurídico, el universalismo de los derechos humanos, la desterritorialización de la producción y aplicación jurídica, la armonización de diferentes órdenes jurídicos, la cesión de soberanía en beneficio de órganos y arreglos supranacionales y la apertura hacia el derecho privado de las transacciones. En la revisión del estado del arte se destacó el papel realizado por los tribunales internacionales que han dado un impulso unificador y fertilizador para el derecho constitucional, a través de la generación de precedentes (Groppi, 2012).

En las entrevistas se preguntó acerca de la opinión sobre los organismos de carácter trasnacional que ejercen jurisdicción o funciones cedidas. Los entrevistados consideraron que el Estadonación se debilita con la globalización al ceder soberanía, aceptar intervención externa y funcionar de manera interdependiente entre países y regiones; algunas posibilidades de mitigar esta tendencia es realizar procesos o acuerdos de integración entre países.

\subsection{CAMBIOS EN EL DERECHO CONSTITUCIONAL, COMO RESULTADO DEL PROCESO DE GLOBALIZACIÓN}

Parecieran confluir dos grandes tendencias globalizadoras con disímil resultado para el constitucionalismo: la dinámica económica señala la internacionalización de las relaciones y transacciones mercantiles y la globalización del capital; este movimiento afecta a todas las esferas del derecho (especialmente potencia el derecho comercial internacional), pero en el caso del derecho constitucional debilita la constitución nacional que está asociada a la idea o concepto del Estado social de corte garantista (Maestro, 2001). Por otro lado, la universalización de los derechos humanos ha elevado a un rango normativo internacional el tratamiento de estos temas y ha fortalecido el constitucionalismo a nivel global, al generar una cultura y lenguaje propio de los derechos humanos, ampliamente compartida ente los tribunales y jueces internacionales (Fuentes, 2010).

Sobre el primer punto, al revisar el estado del arte se encontró que en la medida en que la globalización conlleva la cesión de competencias y funciones a favor de organizaciones internacionales y globales, la constitución, como norma normarum, ya no es la única de carácter superior y, por tanto, debe compartir y admitir el derecho producido por nuevas autoridades de orden supraestatal. Esto se comprueba especialmente en la remisión que la constitución hace hacia tratados y convenios de DDHH, así como la posibilidad de conformar organizaciones supraestatales de integración, que reciben la cesión de soberanía ${ }^{5}$.

5. La Constitución Política de Colombia, en sus artículos 53, 93 y214, realiza la remisión a convenios de la OIT, tratados de DDHH y el DIH respectivamente, como parte del bloque de constitucionalidad. El artículo 150, num. 16, introduce la posibilidad de traslado de atribuciones y competencias propias del Estado nacional en favor de cierto tipo de organismos supraestatales. 
Por otro lado, la globalización implica el debilitamiento del derecho constitucional que se ha desarrollado bajo los supuestos del Estado nacional de carácter garantista (Gutiérrez, 2002), lo cual se ve reflejado en la renuencia de ciertos tribunales constitucionales, como el alemán, para aceptar la jurisprudencia del TJUE, por ejemplo (De Miguel, 2010). En este sentido, la teoría del Estado, base teórica y doctrinal del derecho constitucional moderno, no encaja en la nueva realidad de ordenamientos supraestatales caracterizados por la movilidad de personas, la circulación acelerada de bienes, servicios, capital e información por todo el orbe (Mendivil, 2010; Tushnet, 2009).

Con relación al segundo punto, la globalización del derecho empezó con la universalización de los derechos humanos, su incorporación en los textos constitucionales y la remisión de la constitución a los tratados sobre DDHH (Álvarez, 1998). Ello significó el desarrollo del constitucionalismo durante la segunda mitad del siglo XX y el fenómeno de la constitucionalización del derecho (Gutiérrez, 2002). En este sentido, la constitución se fortalece como ley fundamental, garante de principios y derechos fundamentales, lo que permite hablar de bloque de fundamentalidad (Duarte, 2010).

Según las entrevistas, las áreas más afectadas del derecho interno por la globalización son el derecho comercial, los derechos humanos, el derecho internacional y luego el derecho penal y laboral; como se observa, los DDHH están en segundo lugar, lo que significa un campo muy sensible a los procesos globalizadores.

\subsection{CAMBIOS EN EL DERECHO ADMINISTRATIVO COMO RESULTADO DEL PROCESO DE GLOBALIZACIÓN}

Se pueden identificar dos grandes procesos en los cuales la globalización ha impactado al derecho administrativo: I) La aparición de un derecho administrativo global para dar respuesta, a su vez, al fenómeno de administraciones públicas globales o internacionales; II) El giro dramático del derecho administrativo nacional como resultado de las presiones en los paradigmas de la gestión pública y la organización de la administración pública estatal, con tendencia hacia enfoques de la gestión privada.

Sobre el primer punto, la globalización ha generado el fenómeno de Administraciones sin Estado, esto es, el surgimiento de otras administraciones públicas globales o internacionales que no pertenecen a un Estado en particular y que se concretan en un conjunto de organizaciones internacionales que forman lo que se podría llamar una arquitectura institucional internacional (Fernández, 2005). Estas administraciones tienen su propio personal, presupuesto, normatividad, procedimientos administrativos y, en algunos casos, hasta tribunales propios para dirimir los conflictos con sus funcionarios. Ejemplos de estas administraciones públicas globales son la ONU, con todos sus organismos, el Banco Mundial, la OMC, la INTERPOL, la OEA, ente otras (Brewer-Carías, 2009; Burgos, 2012). 
El derecho administrativo global se concibe como un conjunto de principios, reglas y pactos que surgen a partir de la necesidad de regular situaciones administrativas y de controlar las decisiones de organismos globalizados; es decir, se trata de un control jurídico de la función administrativa de esos órganos y organismos globalizados (Kingsbury, Krisch y Stewart, 2005). En este sentido se habla de sistemas de gobernanza global, en los cuales estas normas administrativas han desempeñado un importante rol. En esta arquitectura institucional globalizada hacen parte, además, las redes de políticas con diversos actores (Estados, ONGs, empresas, organismos internacionales, públicos interesados, personalidades privadas, etc.), los cuales hacen posible la generación, implementación y diseño de políticas públicas.

También se diferencia el derecho administrativo global del derecho administrativo internacional; este último de existencia previa, y que se refiere al control jurídico de la función administrativa estatal que se desarrolla fuera de las fronteras nacionales.

Una conclusión sobre el tema es que el espacio administrativo internacional es diverso y complejo, regulado por diversos sistemas o normas no unificadas aún y con múltiples instituciones regulatorias, en donde ha encontrado gran utilidad el uso del softlaw, muchas veces obligatorio, ya que está conformado por "Preceptos que emanan de organizaciones internacionales que consisten, en cierta forma, en expectativas de conducta, pero que no son de aplicación obligatoria (Fernández, 2005, p.53). Por ello se recomienda la creación de un ejecutivo internacional que facilite la unificación, buscando el beneficio mutuo antes que la imposición.

El segundo punto agrupa discusiones sobre los cambios experimentados por el derecho administrativo estatal en la globalización. Los tratadistas analizan primero las transformaciones de los modelos de gestión y organización pública, para luego determinar los cambios en el derecho administrativo.

Para algunos, los cambios experimentados por la administración pública son entre otros: I) La privatización de funciones a favor del mercado; II) La desregulación de ciertos campos para facilitar la flexibilización, la apertura económica y la competitividad; III) La descentralización territorial, que potencia los territorios, pero que conlleva a la desjerarquización del aparato estatal, IV) El cambio en los modelos de gestión e intervención desde el modelo burocrático tradicional hacia modelos de Nueva Gestión Pública - NGP, que tratan de acercar las prácticas de la gestión pública a las prácticas de la administración de negocios (Restrepo y Rodríguez, 2010).

Sobre el último asunto, algunos señalan que la globalización ha impuesto como estándar la teoría del Buen gobierno, cuyo efecto práctico es reducir la autonomía del Estado en la producción de políticas públicas, pues la mayoría de ellas están redireccionadas desde organizaciones y organismos internacionales (Rincón, 2004).

Por su parte, los entrevistados señalaron que el derecho interno está influido por el derecho globalizado y, en este sentido, deberá ajustarse a dichas disposiciones. Los académicos 
son más puntuales al señalar los condicionantes para el derecho interno: hibridación, armonización y multiculturalidad.

Finalmente, entre las áreas del derecho administrativo afectadas directamente por la globalización se señalan:

- $\quad$ La contratación pública (afectada por principios y prácticas del derecho privado).

- El empleo público (introducción de cargos directivos de "gerencia pública" y tendencia a la valoración del desempeño por productividad).

- El servicio a los ciudadanos, con nuevas modalidades de prestación de los servicios mediante alianzas público-privadas, contratos-plan, creación de fondos especiales, etc.

- En la organización estatal, la creación de nuevos tipos de organismos como las "agencias" (Restrepo y Rodríguez, 2010).

\section{CONCLUSIONES}

El estudio realizado permite concluir que los efectos o consecuencias de la globalización del derecho en la relación Estado-territorio, presenta el siguiente panorama:

De los tres enfoques o teorías que explican la relación Estado y territorio, la más afectada es la teoría acerca de que el territorio es un ámbito físico para el ejercicio de la competencia jurídica; como se dijo, esto influye en el principio de territorialidad de la ley, el cual queda modificado con amplias excepciones. La aceptación de ordenamientos jurídicos externos y el reconocimiento de otras jurisdicciones, autoridades, jueces y tribunales son un indicador incuestionable de tal situación; se destaca el papel de los precedentes y de la jurisprudencia de tribunales internacionales como el TJCA y el TJUE, que son relevantes dentro del derecho interno.

Sin embargo, se evidencia un reforzamiento de la segunda teoría sobre el papel del territorio para el Estado, es decir, el territorio es y seguirá siendo entendido como un elemento esencial del Estado, pese a la globalización. Por ello se exacerban los principios de impenetrabilidad e inviolabilidad del territorio. En las entrevistas se coincide en que el territorio seguirá siendo parte esencial del Estado; los académicos señalan los cambios en la relación ciudadano-territorio y la tendencia a la virtualización de los espacios físicos; los funcionarios, están más preocupados por la relación entre soberanía y territorio, en especial, las repercusiones de una soberanía transformada sobre el concepto de territorio.

Lo anterior significa dos consecuencias adicionales para la soberanía (concepto consustancial a la idea de Estado-nación): a) Un debilitamiento de la soberanía en su sentido general o 
competencial, es decir, como verdadera capacidad y libertad de un Estado y un pueblo para determinar sus políticas públicas; b) Un fortalecimiento de la soberanía en su sentido específico o aspecto territorial, lo cual se concreta en la defensa a ultranza del territorio por acciones de Estados cercanos o lejanos. Por ello, se puede decir que la soberanía ha quedado reducida a su elemento geográfico o territorial.

De otra parte, los impactos de la globalización en el Estado-nación invitan a proponer lo siguiente: a) Debilidad del Estado-nación, en cuanto a crisis de los principios constitucionales tradicionales, la transformación de los elementos clásicos del Estado y la aparición de organizaciones supraestatales y transnacionales; el Estado ha perdido poder y monopolio tanto en la producción jurídica como en el ejercicio de la coacción física. b) Necesariedad del Estado, la crisis económica mundial de los últimos años ha demostrado la vigencia del Estado como único actor capaz de intervenir en la economía e implementar políticas tendientes a la reactivación; por otro lado, el sistema mundial de gobierno aún acusa debilidad, falta de coordinación y liderazgo legítimo para enfrentar los desafíos de regulación y control de los problemas globales; finalmente, el Estado continúa desempeñando importantes funciones en cuanto a provisión y regulación de servicios públicos esenciales. Antes que el final del Estadonación, se debe considerar su transformación y ajuste. Finalmente, Existen, al menos, tres posibilidades sobre el papel del Estado en el futuro inmediato, a saber:

Estado-bisagra y constitución débil. Una mirada pesimista que toma como punto de partida los diagnósticos realizados sobre las crisis de los principios constitucionales, lleva a plantear que el Estado cumplirá una labor al servicio de los intereses que se benefician con la globalización. El Estado será utilizado para sofocar tensiones y resistencias que produce la estrategia de acumulación del capital financiero; por ello no es de extrañar que el Estado pueda, incluso, aumentar su poder de intervención en un sistema en el cual se necesite la articulación de los diferentes niveles de regulación e intervención local y global.

Estado autárquico antisistema. Comprendería ciertos países que se retiran hacia el nacionalismo y reafirman la independencia y la soberanía del Estado. Pueden estar conducidos por regímenes políticos poco democráticos, cerrados, populistas y autoritarios. La posición antisistema los blinda de posibles intromisiones en el manejo de temas domésticos, aunque en materia de derechos humanos, armas nucleares y otros aspectos pueden ser cuestionados por la comunidad internacional. Como estrategia geopolítica, estos Estados pueden, a su vez, realizar convenios y alianzas internacionales con otros que están en la misma situación.

Estado social de derecho y constitución fuerte. Esta mirada combina las oportunidades que puede ofrecer la globalización para el logro de los fines del Estado y la pervivencia del Estado constitucional garantista. Frente a la globalización, se trataría de una propuesta para cimentar un cosmopolitismo en defensa de la herencia común de la humanidad, como son los temas de los $\mathrm{DDHH}$, medio ambiente y migraciones, entre otros. 


\section{REFERENCIAS}

- Agnew, J. (2008). Spatiality and territoriality in contemporary social science. En Piazzini, C. \& Montoya, V. (eds.). Geopolíticas: espacios de poder y poder de los espacios. Medellín: La Carreta Editores, 15-29.

- Álvarez, L. (1998). Globalización, integración y derecho constitucional. En Memorias II Jornadas Chileno-Argentinas de Derecho Constitucional, 467-483. Recuperado de: http:// www.juridicas.unam.mx/publica/librev/rev/dconstla/cont/2003/pr/pr26.pdf. Consultado el 15.4.2012.

- Balaguer, F. (2007). Los tribunales constitucionales en el proceso de integración europea. Revista de Derecho Constitucional Europeo, 4(7), 327-378. Recuperado de: http://www.ugr. es/ redce/REDCE7/articulos/12franciscobalaguercallejon.htm. Consultado el 18.10.2012.

- Bell, D. (2000).Un mundo desbocado. Los efectos de la globalización en nuestras vidas. Madrid: Taurus.

- Blanco, C. (2012). Las manifestaciones de voluntad del órgano deliberante de la Comunidad Andina: ¿Utopía o realidad? Bogotá: Universidad Libre.

- Borja J. \& Castells, M. (1998). Local y global. La gestión de las ciudades en la era de la información. Madrid: Taurus.

- Brewer-Carías, A. (2009). El derecho administrativo global en materia de cooperación policial y el procedimiento administrativo global desarrollado ante INTERPOL, como administración global, para la protección de las personas. Ponencia llevada a cabo en el Tercer Congreso Internacional de Derecho Administrativo, Monterrey, UNAM y Universidad Autónoma de Nuevo León. Recuperado de : http://www.allanbrewercarias. com/Content/449725d9-f1cb-474b-8ab2-41 efb849fea2/Content/l,\%201,\%20990.\%20 PROCEDIMIENTO\%20ADMINISTRATIVO\%20GLOBAL\%20EN\%20MATERIA\%20DE\%20 POLIC\%C3\%8DA.\%20Monterrey.doc).pdf. Consultada el 10.2012.

- Burgos, G. (2012). Conflictos comerciales internacionales. El acceso al mecanismo de solución de controversias de la OMC. Bogotá: Universidad Libre.

- De Blas, A. (1981). Elementos constitutivos del Estado. En García, R. (comp.). Introducción a la teoría del Estado. Barcelona: Editorial Teide, 75-101.

- De Miguel, J. (2010). La justicia constitucional en la teoría de la constitución europea. En Bazán, V. Derecho Procesal Constitucional Americano y Europeo. Buenos Aires: Editorial Abeledo Perrot, Tomo II, 1535-1560. 
- Duarte, M.L. (2010). União Europeia y Proteção dos Dereitos Fundamentais. Uma Justiça Constitucional de Base Triangular. En Bazán, V. Derecho Procesal Constitucional Americano y Europeo. Buenos Aires: Editorial Abeledo Perrot, 1598-1616.

- Fernández, P. (2005). Globalización y derecho público. Introducción al derecho administrativo internacional. En Cienfuegos, D. y López, A (coords.). Estudios en homenaje a don Jorge Fernández Ruiz. México: Instituto de Investigaciones Jurídicas de la Universidad Nacional Autónoma de México, Tomo I: Derecho administrativo, 45-63. Recuperado de: http://biblio.juridicas.unam.mx/libros/4/1594/7.pdf. Consultado el 5.6.2012.

- Fuentes, R.E. (2010). La globalización y su impacto en el derecho constitucional (tesis doctoral). Barcelona: Universidad Autónoma de Barcelona. Recuperado de: www. tesisenred.net/handle/10803/32074. Consultada en 6.2012.

- Groppi, T. (2010). ¿Bottom up globalization? El uso de precedentes extranjeros por parte de las cortes constitucionales. En Diritti Comparati, 1-5. Recuperado de: http://www. diritticomparati.it/2010/12/bottom-up-globalization-.html. Consultado el 12.9.2012.

- Gutiérrez, I. (2002). Globalización, Estado y derecho constitucional. A Distancia, 19(2), 17-21.

- Herrero, M. (1983). La estructura jurídico-política del Estado constitucional. En Alzaga, O. et. al. Derecho Político (I). Madrid: UNED, 95-114.

- Jaeger-Fine, D. et. al. (2013). Internet Jurisdiction: A Survey of German Scholarship and Cases, In Forham Law School, Fordham Center on Law and Information Policy. Recuperado de: http://ssrn.com/abstract=2309575. Consultado el 12.12.2013.

- $\quad$ Kingsbury, B.; Krisch, N. \& Stewart, R. (2005). The Emergence of Global Administrative Law. 68 Law and Contemporary Problems-(GAL) Project, 15, 15-61. Recuperado de: http:// iilj.org/GAL/documents/TheEmergenceofGlobalAdministrativeLaw.pdf. Consultado el 21.10.2012.

- Kohl, U. (2010). Jurisdiction and the internet regulatory competence over online activity. Second edition, Cambridge University Press, Cambridge Book Online.

- Maestro, G. (2001). Globalización y constitución débil. En Teoría y Realidad Constitucional, 7, 137-152. Recuperado de: http://www.juridicas.unam.mx/publica/librev/rev/trcons/ cont/7/est/est5.pdf. Consultado el 9.9.2012.

- Manrique J. (2011). The Assertion of Judicial Jurisdiction over Cyber-Torts. A Comparative Analysis. Revista de Derecho, Comunicaciones y Nuevas Tecnologías, 5, 2-24. 
- Martínez, J. (2010). Y todo en tu nombre. En Hernández, C.A. (comp.). Globalización y derecho. Bogotá: Universidad Libre, 157-175.

- Mendivil, P. (2010). El concepto de Constitución en el marco de la globalización del derecho. En Hernández, C.A. (comp.). Globalización y derecho. Bogotá: Universidad Libre, 207-216.

- Naranjo, V. (2003). Teoría constitucional e instituciones políticas. Novena edición, Bogotá: Temis.

- Palomares, J.R. (2007). Comunidad Andina: ¿Es vinculante el derecho comunitario? Revista Virtual Via Inveniendi et iudicandi, sin paginar, 17 páginas, en http://www.usta. edu.co/progamas/derecho/revistainveniendi/revista/imgs/HTML/revistavirtual. Consultado el 28.5.2012.

- Ramírez, G. (s. f.). Transformaciones del constitucionalismo en el contexto de la globalización. Universidad Externado de Colombia, 35 páginas. Recuperado de: http://icr.uexternado. edu.co/Documentos/ponencia2.pdf. Consultado el 3.9.2010.

- $\quad$ Reindenberg, J. et al (2013). Internet Jurisdiction: A Survey of Legal Scholarship published in English and United States cases Law. At Forham Law School, Fordham Center on Law and Information Policy. Recuperado de: http://ssrn.com/abstract=2309526. Consultado el 6.12.2013.

- Restrepo, M.; Rodríguez, J. et al (2010). Globalización del Derecho Administrativo colombiano. Bogotá: Ediciones Universidad del Rosario.

- Rincón, J. (2004). Globalización y derecho administrativo. Bogotá: Pontificia Universidad Javeriana. Recuperado de: www.viewcontent.cgi.pdf. Consultado en 4.2013.

- Santos, B. de S. (2002). La globalización del derecho. Los nuevos caminos de la regulación y la emancipación. Segunda reimpresión, Bogotá: Universidad Nacional-ILSA.

- Svantesson, D. (2007). Private International Law and the Internet. Alphenaan den Rijn, Kluwer Law International.

- Tajadura, J. (2004). ¿El ocaso de Westfalia? Reflexiones en torno a la crisis del constitucionalismo en el contexto de la mundialización. Revista de Estudios Políticos (Nueva Época), 123, 315-349. 
- Tushnet, M. (2009). The inevitable globalization of Constitucional Law. Opinio Juris, 1-2. Recuperado de: http://opiniojuris.org/2009/06/11/the-inevitable-globalization-ofconstitutional-law/. Consultado el 14.9.2012.

- Twining, W. (2003). Derecho y Globalización. Bogotá: Universidad de los Andes.

- VUVrije Universiteit Amsterdam (2013). Boundaries of Law. Research program. Recuperado de: http://www.rechten.vu.nl/en/research/research-programmes/boundariesof-Law/index.asp. Consultado el 13.3.2013.

- Wolkermer, A. (2006). Pluralismo jurídico: fundamentos de una nueva cultura del derecho. Alcalá de Alcadaíra: Editorial MAD, Colección Universitaria de Textos Jurídicos. 